\title{
Performance Analysis of a Cross-Layer Handoff Ordering Scheme in Wireless Networks
}

\author{
Pochiang Lin, Student Member, IEEE, Tsungnan Lin, Senior Member, IEEE, \\ and Chiapin Wang, Member, IEEE
}

\begin{abstract}
In this paper we propose a cross-layer handoff ordering scheme. The frame success rate (FSR) is adopted as the basis of prioritization. Different quality of service (QoS) requirements of various applications would result in different FSR requirements. In order to indicate how critical a handoff request is, both the FSR requirement from the application layer and the FSR measurement from the medium access control layer are taken into consideration in the proposed scheme. The prioritization of handoff requests follows the most-critical-first policy. Performance analysis shows that the proposed scheme effectively reduces the forced termination probabilities. Under the same forced termination probability requirements, it could provide $1.95 \%$ to $11.13 \%$ more arrival calls compared to previous works.
\end{abstract}

Index Terms-Cross-layer approach, handoff ordering, wireless networks.

\section{INTRODUCTION}

W IRELESS bandwidth is one of the most valuable and limited resources. It is often divided into multiple channels. The number of channels allocated to a base station (BS) determines the number of users that can be served simultaneously by this BS. When all channels of a BS are assigned to existing calls, newly arrived calls would be blocked, and handoff calls from other BSs might be forced to terminate. Since terminating an ongoing call, from a user's perspective, is much more unacceptable than blocking a new call, appropriate mechanisms should be provided to reduce the forced termination probability while keeping an acceptable new call blocking probability.

Many solutions were already proposed, e.g. call admission control schemes [1] [2] [3] [4], channel reservation schemes [5] [6] [7], and handoff ordering schemes [8] [9] [10] [11]. While the first two types of schemes would suffer from a lower utilization of channel resources, Narendran et al. proved that the handoff ordering schemes provide more effective channel utilization [9]. In the handoff ordering schemes, the target BS temporarily puts the handoff requests in its queue. A mobile unit (MU) which initiates a request keeps the connection to its original BS and waits for an available channel

Manuscript received July 9, 2007; revised December 17, 2007; accepted April 21, 2008. The associate editor coordinating the review of this paper and approving it for publication was G. Mandyam. This work was supported in part by Yulon-Nissan Motor Co., Taiwan, National Science Council under Grant 96-2221-E-002-091 and Grant 96-2219-E-002-006, and by Excellent Research Projects of National Taiwan University, 97R0062-06.

P. Lin and T. Lin are with the Department of Electrical Engineering and Graduate Institute of Communication Engineering, National Taiwan University, Taipei, 106, Taiwan (e-mail: tsungnan@ntu.edu.tw).

C. Wang was with Graduate Institute of Communication Engineering, National Taiwan University. He is now with the Institute of Applied Electronic Technology, National Taiwan Normal University, Taipei, 106, Taiwan.

Digital Object Identifier 10.1109/T-WC.2008.070747 until the link quality becomes too weak. If the MU still can not get an available channel when its link quality is below the minimum requirement, this call would be forced to terminate. Thus it is important to prioritize the requests based on their link qualities.

The majority of existing handoff ordering schemes adopt the received signal strength (RSS) as the link quality metric [8] [9] [10] [11]. However, RSS can not directly represent the user perceived quality of service (QoS). Many other factors, e.g. noise, interference, frame length, and modulation/coding schemes, would also affect the QoS. Besides, different applications would have different QoS requirements. Since RSS is a measurement at the physical (PHY) layer only, it cannot be easily mapped to various QoS requirements. Therefore, we are motivated to design a cross-layer handoff ordering scheme.

In the proposed scheme, the frame success rate (FSR) at the medium access control (MAC) layer, rather than the RSS at the PHY layer, is adopted as the link quality metric to prioritize the handoff requests [12]. FSR is more suitable to represent the link quality, because it can be easily mapped to the application (APP) layer QoS requirements of various applications, e.g. throughput, delay, and packet success rate. When FSR is below minimum requirement, users would get frustrated with the QoS even when RSS is still acceptable. Lal et al. show that FSR can be measured in an efficient way [13]. They find that only a few measurements of the wireless channel are sufficient to obtain a good estimate of FSR. This finding validates the feasibility of on-line FSR measurement. In our proposed scheme, the priority of a handoff request is determined by its remaining time to reach the minimum FSR requirement, i.e. the most-critical-first policy is followed. Estimation of the remaining time of each handoff request is based on its current FSR, the FSR degradation rate, and the minimum FSR requirement. We analyze the performance of the proposed scheme and compare it with the previous works. Results show that the proposed handoff ordering scheme effectively reduces the forced termination probability, and keeps almost the same new call blocking probability as the previous works. In other words, the proposed scheme could support more arrival calls under the same forced termination probability requirements.

The rest of this paper is organized as follows. In Section II we provide the detailed description of the proposed handoff ordering scheme. Performance analysis is presented in Section III, followed by the numerical results and discussion in Section IV. Finally, the conclusion is given in Section V. 


\section{PROPOSED CROSS-LAYER HANDOFF ORDERING SCHEME}

\section{A. Relationship between FSR and QoS}

First we present the relationship between FSR and QoS by an example. Consider one service class with throughput requirement $T_{r e q}$, delay requirement $D_{\text {req }}$, and packet success rate (PSR) requirement $P S R_{r e q}$. Each packet generated by this service class contains a fixed payload length $L$. A fixed overhead length $H$ consists of the headers and tailers of all layers. Assume that this system adopts the stop-and-wait protocol in the data link layer. The length of acknowledge frame is $A$. The transmission rate is $R$. It is assumed that the transmission of all frames, including data and acknowledge frame, follows the same transmission rate. The propagation delay between the transmitter and the receiver is $D_{p}$. Here we consider the case that the payload length $L$ is small enough so that no packet fragmentation occurs. Analysis for the case of packet fragmentation could also be obtained by similar processes. In accordance with the throughput requirement $T_{\text {req }}$, the throughput $T$ has to meet the following equation.

$$
T=\frac{L}{\left(\frac{1}{F S R}\right)\left(2 D_{p}+\frac{H+L+A}{R}\right)} \geq T_{r e q}
$$

Thus the frame success rate has to meet the following equation.

$$
F S R \geq \frac{T_{r e q}\left(2 D_{p}+\frac{H+L+A}{R}\right)}{L}=F S R_{\min 1}
$$

Then we consider the delay requirement $D_{\text {req }}$. The delay $D$ has to meet the following equation.

$$
\begin{aligned}
D= & \left(\frac{1}{F S R}-1\right)\left(2 D_{p}+\frac{H+L+A}{R}\right) \\
& +D_{p}+\frac{H+L}{R} \\
\leq & D_{\text {req }} .
\end{aligned}
$$

Thus the frame success rate has to meet the following equation.

$$
F S R \geq \frac{2 D_{p}+\frac{H+L+A}{R}}{D_{r e q}+D_{p}+\frac{A}{R}}=F S R_{\min 2} .
$$

Since no packet fragmentation occurs in this case, the frame success rate also has to meet the following equation while considering the PSR requirement.

$$
F S R \geq P S R_{r e q}=F S R_{\min 3} .
$$

Accordingly, in order to meet all requirements, the minimum requirement of frame success rate is set as $F S R_{\min }=$ $\max \left(F S R_{\min 1}, F S R_{\min 2}, F S R_{\min 3}\right)$. The purpose of this example is to illustrate that there would be a relationship between FSR and QoS given the system protocols, mechanisms, and parameters. Under various system protocols, mechanisms, and parameters, there would be certainly different relationship between FSR and QoS.

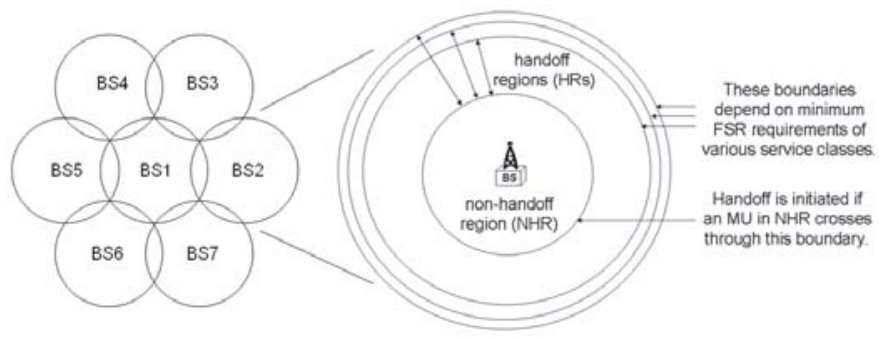

Fig. 1. System model consists of multiple homogeneous cells. Each cell consists of one NHR and multiple HRs for different service classes.

\section{B. Proposed handoff ordering scheme}

The proposed scheme follows the most-critical-first policy. Each MU moving away from its original BS will reach its minimum FSR requirement at some time in the future. The remaining time to reach the minimum FSR requirement is denoted as $t_{r}$. The handoff request with smaller $t_{r}$ is more likely to be terminated sooner due to bad link quality. Thus a higher priority should be assigned to this one in order to reduce the forced termination probability.

Assume that there are $M$ service classes, and the minimum FSR requirement for the $i^{t h}$ service class is $F S R_{\min }(i), i=$ $1,2, \ldots, M$. For the handoff request $j$ in the queue, its minimum FSR requirement is $F S R_{\min }(c(j))$, where $c(j)$ is the service class of request $j$. The currently received FSR of request $j, F S R_{\text {cur }}(j)$, is measured and recorded every $\Delta t$ interval. The FSR degradation rate $s(j)$ is obtained from the current and previous samples of FSR, as (6) shows.

$$
s(j)=\frac{F S R_{\text {pre }}(j)-F S R_{c u r}(j)}{\Delta t}
$$

where $F S R_{\text {pre }}(j)$ is the previous sample of the FSR. The remaining time $t_{r}(j)$ is estimated as (7) shows.

$$
t_{r}(j)=\frac{F S R_{c u r}(j)-F S R_{\min }(c(j))}{s(j)}
$$

In accordance with the most-critical-first policy, the priority of handoff request $j$ is designed as $P(j)=1 / t_{r}(j)$.

\section{PERFORMANCE ANALYSIS}

Chung and $\mathrm{Li}$ provide a performance analysis of first-in first-out (FIFO) handoff ordering scheme [14]. Based on their work, we make some modifications to cover other handoff ordering schemes, including SSMC [11] and the proposed scheme. We consider a wireless communication system which consists of multiple homogeneous cells, as Fig. 1 shows. Each cell is divided into several regions, including one non-handoff region (NHR) and several handoff regions (HRs). The HR for the $i^{\text {th }}$ service class is denoted as $H R_{i}$. The outer bound of $H R_{i}$ depends on its specific minimum FSR requirements. If an MU is located in the NHR, there is no handoff requirement. If this MU keeps on moving and crosses the boundary between NHR and HR, a handoff is initiated. Let $R_{H R, i}$ be the ratio between the HR area and the whole cell area when considering the $i^{t h}$ service class, i.e., $R_{H R_{i}}=A_{H R_{i}} /\left(A_{N H R}+A_{H R_{i}}\right)$, where $A_{H R_{i}}$ and $A_{N H R}$ are the areas of $H R_{i}$ and the NHR, respectively. 
The new call arrival rate is assumed to be Poisson with average arrival rate $\lambda_{n}$. Among the new arrival calls, the call arrival rate ratio for the $i^{t h}$ service class, i.e., the probability that a new arrival call belongs to the $i^{\text {th }}$ service class, is denoted as $r_{i}$. Suppose that the initial location of a new arrival call is uniformly distributed over the whole cell. Therefore, when considering the $i^{\text {th }}$ service class, the new call arrival rate in the HR, denoted as $\lambda_{n, i, H R}$, would be equal to $R_{H R_{i}} \cdot r_{i} \cdot \lambda_{n}$, and the new call arrival rate in NHR, denoted as $\lambda_{n, i, N H R}$, would be equal to $\left(1-R_{H R_{i}}\right) \cdot r_{i} \cdot \lambda_{n}$. The average new call arrival rate in the $\mathrm{HR}, \lambda_{n, H R}$, is equal to $\sum_{i=1}^{M} \lambda_{n, i, H R}$, and the average new call arrival rate in the NHR, $\lambda_{n, N H R}$, is equal to $\sum_{i=1}^{M} \lambda_{n, i, N H R}$. The service time $T_{s, i}$ for the $i^{t h}$ service class is assumed to be exponentially distributed with parameter $1 / \mu_{i}$. Thus the average service time $\overline{T_{s}}$ is equal to $\sum_{i=1}^{M} r_{i} \cdot \overline{T_{s, i}}$, and the average service rate $\mu$ is equal to $1 / \overline{T_{s}}$. The cell dwell time $T_{d}$ is assumed to be exponentially distributed with parameter $1 / \eta$. Chung and Li show that, for a circular cell, the average cell dwell rate $\eta$ is equal to $2 V / \pi R$, where $V$ is the user velocity and $R$ is the cell radius [14]. From their work, the cell dwell time for the $i^{t h}$ service class, denoted as $T_{d, i}$, could be obtained. The average cell dwell time $\overline{T_{d}}$ for the whole system could also be obtained as $\sum_{i=1}^{M} r_{i} \cdot \overline{T_{d, i}}$. Chung and Li also state that the dwell times in the HRs and the NHR are the functions of the average cell dwell time and the area ratio of each region. Following their work, and assuming that the dwell times in $H R_{i}$ and the NHR are also exponentially distributed, and each user walk randomly in the region, the average cell dwell time in each region could be obtained.

$$
\begin{gathered}
\overline{T_{i, H R}}=\frac{1}{\eta_{i, H R}}=16^{\log R_{H R_{i}}} \cdot \overline{T_{d}} . \\
\overline{T_{i, N H R}}=\frac{1}{\eta_{i, N H R}}=2^{\log \left(1-R_{H R_{i}}\right)} \cdot \overline{T_{d}} .
\end{gathered}
$$

The channel holding time $T_{h, i}$ is the minimum of the call service time $T_{s, i}$ and the cell dwell time $T_{d, i}$, i.e., $T_{h, i}=$ $\min \left(T_{s, i}, T_{d, i}\right)$.

The average cell dwell rate in the HR, $\eta_{H R}$, is equal to $\sum_{i=1}^{M} r_{i} \cdot \eta_{i, H R}$, and the average cell dwell rate in the NHR, $\eta_{N H R}$, is equal to $\sum_{i=1}^{M} r_{i} \cdot \eta_{i, N H R}$. When an MU is located in the HR, it may keep moving until it loses connection with the serving BS, or it may move back to the NHR of the original BS. Let $\beta$ be the average moving back probability [14]. Therefore, the handoff departure rate in the $\mathrm{HR}, \eta_{H R, d}$, is equal to $(1-\beta) \cdot \eta_{H R}$, and the region transition rate from the HR back to the NHR, $\eta_{H R, b}$, is equal to $\beta \cdot \eta_{H R}$.

We analyze the performance of handoff ordering schemes by a three dimensional birth-death process. The channel capacity of each cell is denoted as $C$, and the limitation of handoff request queue is denoted as $Q$. Let $\vec{s}$ be the state vector which is defined as follows:

$$
\begin{aligned}
\vec{s}= & \left(N_{H R}, N_{N H R}, N_{Q}\right), \\
& 0 \leq N_{H R}, N_{N H R} \leq C, 0 \leq N_{Q} \leq Q
\end{aligned}
$$

where $N_{H R}, N_{N H R}$, and $N_{Q}$ are the numbers of calls in the HR, NHR, and handoff request queue, respectively. Fig. 2 shows the state transition diagram. The corresponding state

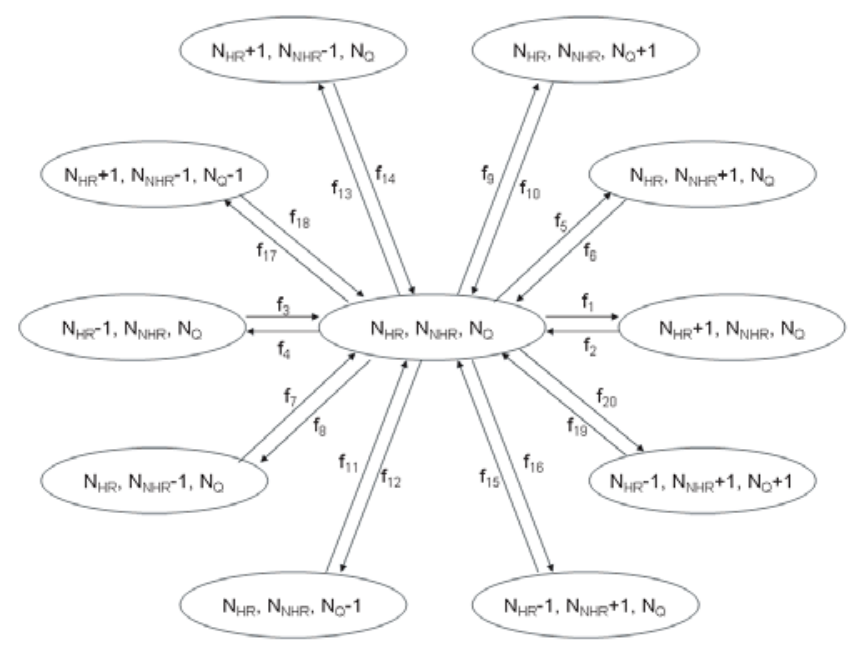

Fig. 2. State transition diagram.

TABLE I

STATE TRANSITION RATES.

\begin{tabular}{c|c}
\hline State Transition Rate & Equation \\
\hline \hline$f_{1}$ & $\lambda_{n, H R}+\lambda_{h}$ \\
\hline$f_{2}$ & $\left(N_{H R}+1\right) \cdot\left(\eta_{H R, d}+\mu\right)$ \\
\hline$f_{3}$ & $\lambda_{n, H R}+\lambda_{h}$ \\
\hline$f_{4}$ & $N_{H R} \cdot\left(\eta_{H R, d}+\mu\right)$ \\
\hline$f_{5}$ & $\lambda_{n, N H R}$ \\
\hline$f_{6}$ & $\left(N_{N H R}+1\right) \cdot \mu$ \\
\hline$f_{7}$ & $\lambda_{n, N H R}$ \\
\hline$f_{8}$ & $\lambda_{N H R}+1 \cdot \mu$ \\
\hline$f_{9}$ & $\lambda_{h}$ \\
\hline$f_{10}$ & $\left.N_{H R} \cdot\left(\eta_{H R, d}+\mu\right)+\left(N_{Q}+1\right) \cdot \mu\right)$ \\
\hline$f_{11}$ & $\left((1-\beta) \cdot \gamma\left(N_{Q}+1\right) \cdot \eta_{H R}+\beta \cdot \eta_{H R}+\mu\right)$ \\
\hline$f_{12}$ & $\lambda_{h}$ \\
\hline$f_{13}$ & $\left(\eta_{H R, d}+\mu\right)+$ \\
\hline$f_{14}$ & $\left((1-\beta) \cdot \gamma\left(N_{Q}\right) \cdot \eta_{H R}+\beta \cdot \eta_{H R}+\mu\right)$ \\
\hline$f_{15}$ & $N_{N H R} \cdot \eta_{N H R}$ \\
\hline$f_{16}$ & $\left(N_{H R}+1\right) \cdot \eta_{H R, b}$ \\
\hline$f_{17}$ & $\left(N_{N H R}+1\right) \cdot \eta_{N H R}$ \\
\hline$f_{18}$ & $N_{H R} \cdot \eta_{H R, b}$ \\
\hline$f_{19}$ & $N_{N H R} \cdot \mu$ \\
\hline$f_{20}$ & 0 \\
\hline
\end{tabular}

transition rates are shown in Table I. The handoff arrival rate $\lambda_{h}$ must satisfy the following equation:

$$
\begin{aligned}
\lambda_{h}= & \left(\sum_{S} N_{N H R} \cdot \eta_{N H R} \cdot p(\vec{s}) \cdot \frac{\eta_{N H R}}{\eta_{N H R}+\mu}\right) \\
& +\left(\lambda_{n, H R} \cdot\left(1-P_{b}\right) \cdot P_{b}\right)
\end{aligned}
$$

where $P_{b}$ is the probability that a new arrival call is blocked from a single cell's point of view. $\gamma\left(N_{Q}\right)$ is used to model the effect on the handoff departure rate for different handoff ordering schemes, which would be explained in the following paragraphs. Let $P=[p(\vec{s})]$ be the state probability vector, and $\Phi$ be the transition rate matrix mentioned above. With $P \Phi=0$ and $\sum_{S} p(\vec{s})=1$, the steady state probability could be obtained. 
On average, each request takes $1 /\left(\eta_{H R}+\mu\right)$ to be taken out from the queue. Assume that there are already $(q-1)$ requests in the queue when the $q^{t h}$ request arrives. First we consider the FIFO scheme. It is obvious that the original $(q-1)$ requests have higher priority than the $q^{\text {th }}$ request. The necessary waiting time is $(q-1) /\left(\eta_{H R}+\mu\right)$. The probability that the handoff departure time is shorter than the necessary waiting time is

$$
P_{F I F O}(q)=1-e^{-\eta_{H R, d} \cdot \frac{q-1}{\eta_{H R}+\mu}} .
$$

Considering all $q$ requests, the effect on handoff departure rate is defined as

$$
\gamma_{F I F O}(q)=\frac{1}{q} \sum_{j=1}^{q} \sum_{i=1}^{M}\left[r_{i} \cdot P_{F I F O i}(j)\right] .
$$

Then we consider SSMC scheme. Assume that there are $i$ out of the $(q-1)$ requests have higher priority. From simulation results it is found that the distribution of priorities by SSMC scheme resembles the exponential distribution. Assume that the priorities for the $m^{\text {th }}$ service class is exponentially distributed with parameter $\lambda_{m}$. The corresponding probability density function and cumulative distribution function are $f_{m}(x)$ and $F_{m}(x)$, respectively. The probability of $i$ is

$$
\begin{aligned}
P(i)= & C_{i}^{q-1} \cdot \int_{t=0}^{\infty} \sum_{m=1}^{M} r_{m} \cdot f_{m}(t) \\
& \cdot\left(\sum_{m=1}^{M} r_{m} \cdot\left(1-F_{m}(t)\right)\right)^{i} \\
& \cdot\left(\sum_{m=1}^{M} r_{m} \cdot F_{m}(t)\right)^{q-i-1} d t .
\end{aligned}
$$

The necessary waiting time for the SSMC scheme is $E[i] /\left(\eta_{H R}+\mu\right)$. Hence, the probability that the handoff departure time of this new arrival request is smaller than the necessary waiting time is

$$
P_{S S M C}(q)=1-e^{-\eta_{H R, d} \cdot\left(\frac{E[i]}{\eta_{H R}+\mu}+\frac{1}{\left(\mu+\eta_{H R, d}\right) \cdot C}\right)} .
$$

Considering all $q$ requests, the effect on handoff departure rate is defined as

$$
\begin{aligned}
\gamma_{S S M C}(q)= & \sum_{j=0}^{q} \frac{j}{q} \cdot C_{j}^{q} \cdot\left(\sum_{i} r_{i} \cdot P_{S S M C, i}(j)\right)^{j} \\
& \cdot\left(\sum_{i} r_{i} \cdot P_{S S M C, i}(j)\right)^{q-j} \cdot
\end{aligned}
$$

Finally we consider our proposed scheme. The probability of $i$ is

$$
P(i)=C_{i}^{q-1} \cdot\left(1-e^{-\eta_{H R, d} \cdot \overline{T_{H R, d}}}\right)^{i} \cdot\left(e^{-\eta_{H R, d} \cdot \overline{T_{H R, d}}}\right)^{q-1-i} .
$$

The probability that the handoff departure time of this new arrival request is smaller than the necessary waiting time is

$$
P_{\text {proposed }}(q)=1-e^{-\eta_{H R, d} \cdot\left(\frac{E[i]}{\eta_{H R}+\mu}+\frac{1}{\left(\mu+\eta_{H R, d}\right) \cdot C}\right)} .
$$

Considering all $q$ requests, the effect on handoff departure rate is defined as

$$
\begin{aligned}
\gamma_{\text {proposed }}(q)= & \sum_{j=0}^{q} \frac{j}{q} \cdot C_{j}^{q} \cdot\left(\sum_{i} r_{i} \cdot P_{\text {proposed }, i}(j)\right)^{j} \\
& \cdot\left(\sum_{i} r_{i} \cdot P_{\text {proposed }, i}(j)\right)^{q-j}
\end{aligned}
$$

When considering the performance of the $i^{\text {th }}$ service classes, the $\eta_{H R, d}$ in (12), (15), and (18) is replaced by $\eta_{i, H R, d}$, and the corresponding $\gamma(q)$ could be obtained.

We evaluate the performance of various schemes based on two metrics: new call blocking probability and forced termination probability. The probability that an arrival call in the cell would be blocked is

$$
P_{b}=\sum_{S_{0}} p(\vec{s})
$$

where $S_{0}=\left\{\vec{s}: \vec{s}=\left(N_{H R}, N_{N H R}, N_{Q}\right) \mid N_{H R}+N_{N H R}=\right.$ $\left.C, 0 \leq N_{Q} \leq Q\right\}$. The new call blocking probability is

$$
P_{b l o c k, i}=R_{H R_{i}} \cdot P_{b}^{2}+\left(1-R_{H R_{i}}\right) \cdot P_{b} .
$$

There are two reasons that would cause the handoff to be failed: the handoff request queue is full, or the handoff departure time expires. The probability that the handoff request queue is full is:

$$
P_{h f 1}=\sum_{\left.\begin{array}{c}
N_{H R}+N_{N H R}=C \\
N_{Q}=Q
\end{array}\right\}} p(\vec{s}),
$$

and the probability that the handoff departure time expires is:

$$
P_{i, h f 2}=\sum_{\left.\begin{array}{c}
N_{H R}+N_{N H R}=C \\
1 \leq N_{Q} \leq Q
\end{array}\right\}}(1-\beta) \cdot \gamma_{i}(q) \cdot p(\vec{s}) .
$$

The handoff failure probability could be written as

$$
P_{i, h f}=P_{h f 1}+\left(1-P_{h f 1}\right) \cdot P_{i, h f 2} .
$$

Forced termination probability considers the fact that a call may meet multiple handoff processes during its whole life time. The handoff probability can be written as

$P_{i, \text { handoff }}=\left(1-R_{H R_{i}}\right) \cdot \frac{\eta_{i, N H R}}{\eta_{i, N H R}+\mu}+R_{H R_{i}} \cdot P_{b} \cdot\left(1-P_{b}\right)$.

With (24) and (25), the forced termination probability can be found as follows.

$$
P_{i, f t}=\sum_{j=1}^{\infty} P_{\text {handoff }}^{j} \cdot\left(1-P_{i, h f}\right)^{j-1} \cdot P_{i, h f} .
$$




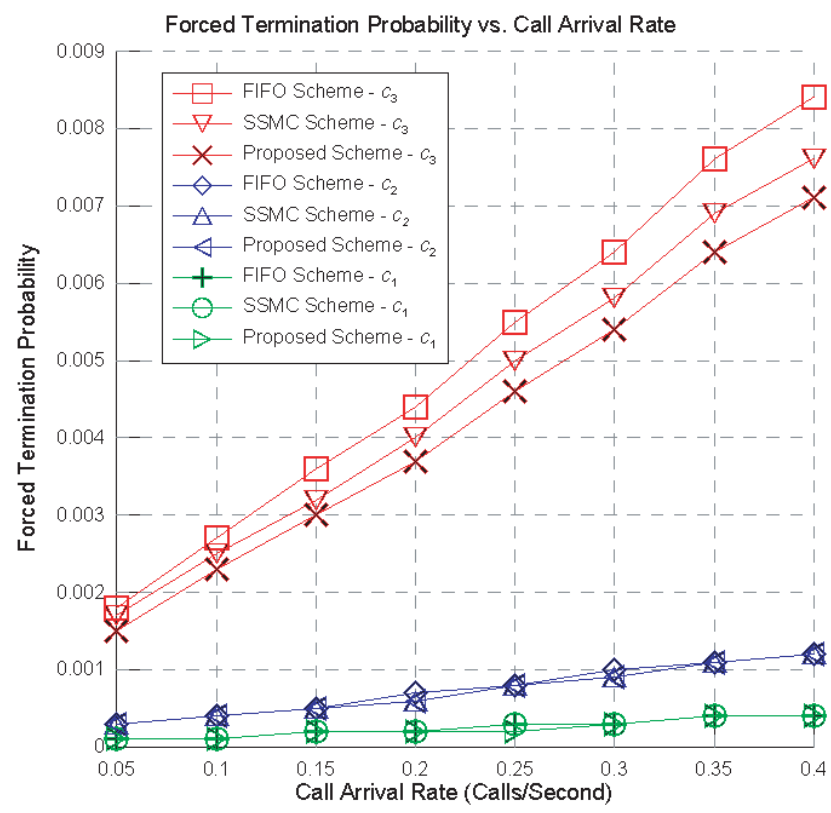

Fig. 3. Forced termination probability vs. call arrival rate. Our proposed scheme outperforms other schemes for the service class $c_{3}$. For the other service classes, the proposed scheme performs a little better than or equal to the other schemes, although the improvement is insignificant in comparison.

\section{NumeriCAL RESUlts AND DISCUSSION}

We compare our proposed scheme with other schemes including FIFO and SSMC. Assume that there are 50 channels allocated to a single BS. The handoff queue size is set as 6. The user moving velocity is assumed to be uniformly distributed between 0 and $90 \mathrm{~km} / \mathrm{h}$. There are three service classes in this system, denoted as $c_{1}, c_{2}$, and $c_{3}$, respectively. The FSR requirements of these three service classes are set as $0.8,0.7$, and 0.9 , respectively. The mean duration time of these three service classes are set as $60 \mathrm{~s}, 300 \mathrm{~s}$, and $900 \mathrm{~s}$, respectively. The new call arrival rate varies from 0.05 to 0.4 calls per second. The arrival rate ratio of the three service classes is set as 40:10:1, i.e. $c_{1}$ is the most popular class. The radius for $N H R, H R_{1}, H R_{2}$, and $H R_{3}$ are set as $2830 \mathrm{~m}$, $3350 \mathrm{~m}, 3630 \mathrm{~m}$, and $2920 \mathrm{~m}$, respectively. The average moving back probability from HR back to NHR is assumed to be 0.37 , as [14] shows.

Fig. 3 shows the forced termination probabilities for $c_{1}, c_{2}$, and $c_{3}$, respectively. Our proposed scheme outperforms other schemes for the service class $c_{3}$, which has the highest FSR requirement. As the call arrival rate increases, the improvement becomes more significant. For the other service classes, the proposed scheme performs a little better than or equal to the other schemes, although the improvement is insignificant in comparison. Fig. 4 shows the relationship between new call blocking probability and call arrival rate. The new call blocking probabilities for all schemes are almost the same, and all increase with the call arrival rate. Therefore, our proposed scheme can really improve the forced termination probability, with almost equal new call blocking probability to the previous works.

Based on these numerical results, the supported call arrival rates under the various requirements of forced termination probabilities are obtained. We take some samples of the

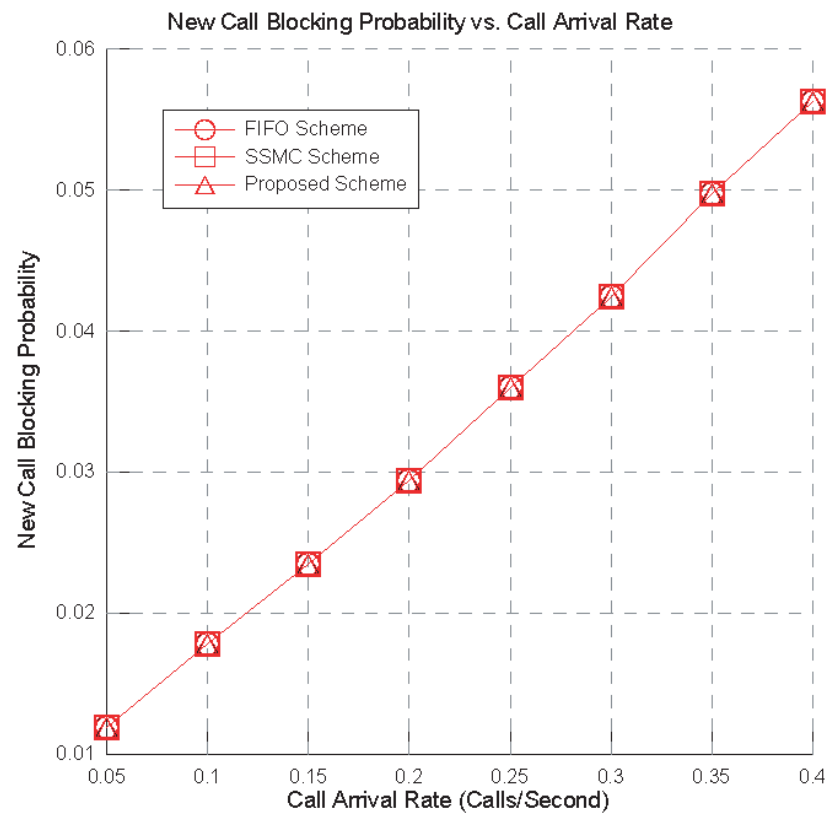

Fig. 4. New call blocking probability vs. call arrival rate. The new call blocking probabilities for all schemes are almost the same.

TABLE II

SUPPORTED CALL ARRIVAL RATE UNDER VARIOUS REQUIREMENTS OF FORCED TERMINATION PROBABILITIES.

\begin{tabular}{c|c|c|c|c}
\hline Service class & $P_{f t}$ Req. & SSMC & Proposed & Improvement (\%) \\
\hline \hline$c_{1}$ & $1.5 E-4$ & 0.1400 & 0.1458 & 4.19 \\
\hline$c_{1}$ & $2.5 E-4$ & 0.2461 & 0.2536 & 3.06 \\
\hline$c_{1}$ & $3.5 E-4$ & 0.3383 & 0.3467 & 2.49 \\
\hline \hline$c_{2}$ & $6.0 E-4$ & 0.1859 & 0.1912 & 2.84 \\
\hline$c_{2}$ & $8.0 E-4$ & 0.2539 & 0.2607 & 2.67 \\
\hline$c_{2}$ & $10.0 E-4$ & 0.3237 & 0.3301 & 1.95 \\
\hline \hline$c_{3}$ & $3.0 E-3$ & 0.1338 & 0.1487 & 11.13 \\
\hline$c_{3}$ & $5.0 E-3$ & 0.2509 & 0.2738 & 9.14 \\
\hline$c_{3}$ & $7.0 E-3$ & 0.3599 & 0.3961 & 10.03 \\
\hline
\end{tabular}

forced termination probability requirements and summarize the results in Table II. The supported call arrival rates of the proposed scheme are always better than those of the SSMC scheme. The percentage of improvement ranges from $1.95 \%$ to $11.13 \%$. The improvement is especially significant for $c_{3}$, which might be the most critical service class since it has the strictest FSR requirement and the longest mean call duration.

\section{CONCLUSION}

While traditional handoff ordering schemes adopt RSS as the basis to prioritize handoff requests, we find that FSR is more suitable for handoff ordering. In this paper we propose a cross-layer handoff ordering scheme based on FSR. The proposed scheme predicts the remaining time for each MU to reach the minimum FSR requirement. The priority of each handoff request is derived from the remaining time. The major contribution of our proposed scheme is that it jointly considers multiple service classes and reduces their forced termination probabilities. This scheme could help the operators to serve more concurrent users to increase revenue while providing satisfactory QoS. 


\section{REFERENCES}

[1] J. Vazquez-Avila, F. A. C. Cruz-Perez, and L. Ortigoza-Guerrero, "Performance analysis of fractional guard channel policies in mobile cellular networks," IEEE Trans. Wireless Commun., vol. 5, no. 2, pp. 301-305, Feb. 2006.

[2] W. Li and X. Chao, "Call admission control for an adaptive heterogeneous multimedia mobile network," IEEE Trans. Wireless Commun., vol. 6 , no. 2, pp. 515-525, Feb. 2007

[3] L. Wang and W. Zhuang, "A call admission control scheme for packet data in CDMA cellular communications," IEEE Trans. Wireless Commun., vol. 5, no. 2, pp. 406-416, Feb. 2006.

[4] E. S. El-Alfy, Y.-D. Yao, and H. Heffes, "A learning approach for prioritized handoff channel allocation in mobile multimedia networks," IEEE Trans. Wireless Commun., vol. 5, no. 7, pp. 1651-1660, July 2006.

[5] Y. Xu, Q. Ding, and C. C. Ko, "Impact of handoff protection strategies on cellular mobile system capacity," IEEE Trans. Wireless Commun., vol. 3, no. 4, pp. 1076-1087, July 2004.

[6] X. Zhu, L. Shen, and T.-S. P. Yum, "Analysis of cognitive radio spectrum access with optimal channel reservation," IEEE Commun. Lett., vol. 11, no. 4, pp. 304-306, Apr. 2007.

[7] J. Roberto, B. de Marca, and L. B. Priolli, "Organizing reserve channels for superior admission control performance," IEEE Trans. Wireless Commun., vol. 5, no. 1, pp. 52-56, Jan. 2006.
[8] S. Tekinary and B. Jabbari, "A measurement based prioritization scheme for handovers in mobile cellular networks," IEEE J. Select. Areas Commun., vol. 10, no. 8, pp. 1343-1350, Oct. 1992.

[9] B. Narendran, P. Agrawal, and D. K. Anvekar, "Minimizing cellular handover failures without channel utilization loss," in Proc. Global Telecommunications Conference (GLOBECOM '94), vol. 3, 1994, pp. $1679-1685$

[10] H. G. Ebersman and O. K. Tonguz, "Handoff ordering using signal prediction priority queuing in personal communication systems," IEEE Trans. Veh. Technol., vol. 48, no. 1, pp. 20-35, Jan. 1999.

[11] R. Chang and S. Leu, "Handoff ordering using signal strength for multimedia communications in wireless networks," IEEE Trans. Wireless Commun., vol. 3, no. 5, pp. 1526-1532, Sept. 2004.

[12] T.-N. L. P.-C. Lin, "Hndoff ordering using link quality estimator for multimedia communications in wireless networks," in Proc. Global Telecommunications Conference (GLOBECOM '05), vol. 2, Nov. 2005.

[13] D. Lal, A. Manjeshwar, F. Herrmann, E. Uysal-Biyikoglu, and A. Keshavarzian, "Measurement and characterization of link quality metrics in energy constrained wireless sensor networks," in Proc. Global Telecommunications Conference (GLOBECOM '03), vol. 1, 2003, pp. 446-452.

[14] S. Chung and M. Li, "Performance evaluation of hierarchical cellular cdma networks with soft handoff queueing," IEEE Trans. Veh. Technol., vol. 54, no. 2, pp. 652-672, Mar. 2005. 